\title{
Numerical effects on energy distribution functions in particle-in-cell simulations with Monte Carlo collisions: Choosing for numerical parameters
}

\author{
M. M. Turner \\ School of Physical Sciences and National Centre for Plasma Science and Technology, \\ Dublin City University, Dublin 9, Ireland
}

\begin{abstract}
Particle-in-cell simulations with Monte Carlo collisions are expected to calculate the velocity distribution functions of charged species correctly, even if these distribution functions have exotic features such as gross anisotropy in velocity space, marked departures from a Maxwell-Boltzmann distribution, or failure of the local field approximation. Correct computation of the electron energy distribution function, in particular, is crucial in chemically complex plasmas, where radicals produced by electron impact processes usually have a dominant role. In such cases, accurate calculation of the rate constants for electron impact processes is a major motivation for the use of a kinetic simulation procedure, such as the particle-in-cell method. Like any numerical procedure, the particle-in-cell algorithm has limitations, and one of these limitations is that velocity space diffusion can distort the particle energy distribution functions. This paper presents examples of some conditions where such numerical distortion of particle energy distribution functions is important, and draws conclusions with implications for the choice of numerical parameters for particle-in-cell simulations. In particular, we show that the number of particles per cell that is required varies greatly with the conditions (as much as three orders of magnitude), and can sometimes be very large indeed. We suggest a heuristic for selecting the number of particles per cell, derived from the examples we discuss.
\end{abstract}

PACS numbers: 


\section{Introduction}

Particle-in-cell simulations with Monte Carlo collisions [1, 2, 3] are widely used, most commonly in applications involving plasma discharges. These discharges present some difficulty to most computational and analytical approaches, primarily because the particle distributions can assume complex forms, but also because the coupling between particles and fields can be non-trivial, which can lead to subtle but important physical effects, such as those related to Landau damping, for example [4]. Physical insight has suggested many useful approximations that greatly simplify the treatment of such problems. For example, if velocity space anisotropy is not severe, a spherical harmonic approximation can be used to represent velocity space distribution functions $[5,6]$. If velocity space anisotropy is extreme, on the other hand, a multi-beam approach can be used. These, and other related approximations, can powerfully reduce the complexity of the problem at hand. However, there is a basic difficulty, namely that these useful approximations are rarely uniformly valid. Typically, they hold in some spatial regions, but not in others. Consequently, there arises the notoriously difficult problem of connecting incommensurate approximations.

A common response is to develop a hybrid approach [7] that is based primarily on computing moments of the Boltzmann equation such as density, momentum and energy, but with the refinement that velocity space distributions are calculated by some other procedure, such as solving a simplified Boltzmann equation. This approach can exhibit all the relevant physics at reasonable computational cost. Such hybrid methods are based on complex assumptions and approximations, whose consistency and validity is difficult to demonstrate. Consequently, the level of accuracy associated with a hybrid simulation is difficult to specify.

A particle-in-cell simulation avoids these difficulties by solving the Boltzmann equation directly, so there is no restriction on the form of the velocity distribution functions or on the nature of the coupling between the particles and the fields. Of course, these advantages are bought at considerable computational cost, and are accompanied by some algorithmic limitations. Most notably, the discrete time and space intervals involved in the method are restricted by well-known conditions related to the plasma frequency and the Debye length, respectively. A third numerical parameter is the number of particles per cell. This parameter essentially controls the rate of velocity space diffusion. The question of how these parameters should be chosen was extensively investigated at an early stage in the development of particle-in-cell simulations (see $[1,2]$ and references therein), and these works produced heuristics for selecting time steps and cell sizes that have been extensively used since. They also yielded relationships between the numerical parameters and the rate of velocity space diffusion. These relations are helpful if the aim is to simulate a closed system for a finite amount of time (such as a model with no power input and periodic boundary conditions), because they enable one to compute a relaxation time due to numerical effects that can be made large compared with the duration of the simulation. But their relevance to 
the situation in most discharge simulations is not obvious, because here one wishes typically to computer a stationary state of the system (in some sense) and what then is an acceptable rate of numerical diffusion? There is no obvious characteristic time to compare with. Consequently, the question of what numerical parameters should be deemed acceptable is not easy. Even if the usual heuristics for time step and cell size are accepted, the question of the number of particles remains open. This issue has received surprisingly little attention. Vahedi et al [8] investigated the effect of numerical parameters in the much discussed case of a low-pressure capacitive discharge in argon (see Godyak and Piejak [9] and citations thereof). Their discussion emphasizes the opportunities for using implicit techniques with sub-cycling to increase the time step and cell size, but they say little concerning the choice of the number of particles. One might infer that they believed their simulations to be satisfactorily converged for the largest number of particles-per-cell that they employed. The present author showed in more recent work that this was probably an optimistic view[10]. In fact, one needs both a greater number of particles and Coulomb collisions between electrons to achieve a converged simulation. This work also showed that the velocity space diffusion rate is - surprisingly - also influenced by the frequency of Monte Carlo collisions. Ref. [10] also defines a relaxation time that can be expressed in terms of the three numerical parameters discussed above, but this relaxation time provides little insight into the character of the distortion of the distribution function, or the relationship between the physical parameters and the occurrence of numerical effects.

This discussion shows that previous work has provided little insight into the relationship between the quality of a simulation and the number of particles per cell. In the present paper, we present calculations aimed at providing some such insight into the character of the distortion of the particle distribution functions produced by velocity space diffusion, and illustrating the relationship between these distortions and a key physical parameter, namely the reduced electric field-the ratio of the electric field to the gas density, $E / N$. Under many conditions of practical interest, the reduced electric field is the physically relevant control parameter, but even when this is not so, $E / N$ regulates the electron temperature, and the electron temperature is almost always of central interest. The drift of argument of this paper is thus that the number of particles per cell that is required is primarily a function of the electron temperature. Consequently, the results presented in this paper may be used to form a preliminary view of the number of particles that might be needed in typical applications of particle-in-cell simulations.

\section{Background}

In particle-in-cell simulations, the causes of velocity space diffusion are imprecision in the solution of the field equations, and the graininess induced by the finite number of particles $[1,2]$. These effects introduce a stochastic component into the fields. By convention, this diffusion effect is divided into self-heating, which causes a roughly 
linear increase in the kinetic energy of the particles, and relaxation (sometimes called thermalisation), which causes the particle distribution functions to become more Maxwellian than should be the case. One can define separate characteristic times for these phenomena: The self-heating time $\tau_{H}$, and the relaxation time $\tau_{R}$. In general, these times are complex functions of three numerical parameters, the cell size $\Delta x$, time step $\Delta t$, and the number of particles per cell, $N_{C}$. However, the cell size and time step are usually governed by the well-known conditions

$$
\begin{aligned}
& \omega_{p} \Delta t \lesssim 0.2 \\
& \frac{\lambda_{D}}{\Delta x} \gtrsim 2
\end{aligned}
$$

where $\omega_{p}$ is the plasma frequency, and $\lambda_{D}$ is the Debye length. These accuracy conditions are almost universally applied. $N_{C}$ can then be adjusted to regulate the rate of heating and thermalisation. These effects drive fluxes of particles in velocity space. Such fluxes are also driven by physical effects, such as electric fields and collisions of various kinds. A reasonable accuracy condition is to require the physical fluxes to be large compared with the numerically induced fluxes. If this condition does not hold, then we must expect the particle distribution functions to be significantly shaped by numerical phenomena, which is clearly an undesirable situation. However, in practice, it is not obvious how to demonstrate that these numerical effects are in fact negligible, without investigating the convergence of every simulation as a function of $N_{C}$. The purpose of the present study is carry out such an investigation for conditions relevant to self-sustaining discharges. We have chosen to work with cross sections for helium gas, and to investigate the convergence of the electron energy distribution function as $N_{C}$ is varied. This has been done for a range of values of the reduced electric field, $E / N$, where $E$ is the electric field and $N$ is the neutral gas number density. The reduced electric field values we employed roughly span the range found in a helium positive column. The positive column, although an important test case, is not of universal interest. However, as noted above, $E / N$ essentially regulates the mean electron energy, $\langle\varepsilon\rangle$, and the range of $\langle\varepsilon\rangle$ that occurs in these calculations probably covers any self-sustaining discharge in helium. Therefore, these results provide useful insight for most if not all simulations of plasma discharges.

\section{Simulations}

The simulation strategy is to perform calculations with physical and numerical parameters that are identical, except for the number of particles per cell, $N_{C}$. This procedure has been carried out for a series of values of the reduced electric field, $E / N$, for reasons discussed above. The range of values adopted is from $1 \mathrm{Td}$ to $200 \mathrm{Td}$. We employed a conventional particle-in-cell simulation with Monte Carlo collisions, using one space dimension, Cartesian coordinates, and periodic boundary conditions. A set of cross sections for electron collisions with helium was used [11]. In all cases, the ratio of the electron collision frequency, $\nu_{c}$, to the plasma frequency was $\sim 0.02$. The results depend on this parameter, which is chosen here to be typical for particle-in-cell 
simulations of discharges. Similarly, the cell size and time step were chosen to marginally satisfy eqs. 1 and 2. The total number of particles and the number of cells were varied in order to achieve the desired value of $N_{C}$. In no case was the number of cells less than 32, however, because below this limit the results depend on the number of cells. Presumably, this happens because the stochastic field spectrum is significantly cut off in smaller simulations. In addition to these particle-in-cell calculations, we also carried out similar calculations using a pure Monte Carlo method. This Monte Carlo method in fact is the same as the particle-in-cell simulation, but with the stochastic field component eliminated. The differences that we find between the particle-in-cell calculations and the Monte Carlo calculations are therefore entirely due to the influence of the stochastic field component in the particle-in-cell simulations. For the purposes of this study, we have assumed that if conditions 1 and 2 are uniformly satisfied, then the primary factor influencing the stochastic field component is the number of particles per cell, $N_{C}$. In all these computations, secondary electrons produced by ionization are suppressed (as a matter of convenience only). In this work we are interested in stationary results, but the simulation procedure follows evolution in time. Therefore, an integration time is chosen long enough for a stationary state to be reached, and the results presented were obtained by taking a time average of this stationary state. This averaging, of course, is needed to reduce statistical fluctuation.

\section{Results}

Fig. 1 shows the mean electron energy as a function of $E / N$, for the range of values that we consider. These data show that the mean energy varies from about $0.6 \mathrm{eV}$ to about $20 \mathrm{eV}$. For $E / N \lesssim 10 \mathrm{Td}$, the mean energy is approximately proportional to the electric field. In this regime, inelastic collision processes are not important. For $E / N \gtrsim 10 \mathrm{Td}$, inelastic processes dominate the electron energy balance, and the mean energy has a much weaker dependence on the electric field. A self-sustaining discharge probably cannot exist in the regime where $E / N \lesssim 10 \mathrm{Td}$, because the ionization rate is vanishingly small, but it is not irrelevant to consider such values. A breakdown of spatial or temporal equilibrium between the electrons and the fields can lead to the appearance of groups of electrons with mean energies of this order $[9,12]$, and an accurate representation of such electrons in simulations can be important.

Fig. 2 shows the electron mean energy at $200 \mathrm{Td}$, calculated using the particlein-cell simulation for a range of values of $N_{C}$. In this case, there is no appreciable dependence of $\langle\varepsilon\rangle$ on $N_{C}$, which, of course, is a desirable situation. The electron energy distribution functions displayed in fig. 3 similarly show that over the entire energy range, there is no important variation with $N_{C}$. In the extreme tail of the distribution, there is indeed some divergence when $N_{C}<10$, but this is a smaller number of particles than one would normally employ in a practical simulation.

Fig. 2 shows the electron mean energy as a function of $N_{C}$ for $E / N=100 \mathrm{Td}$. These data show that for $N_{C} \gtrsim 100$, there is essentially no dependence. For smaller values, a 
weak dependence appears, and the mean energy falls, by about $1 \mathrm{eV}$ between $N_{C}=100$ and $N_{C}=10$. This reflects an underlying distortion of the energy distribution function, which becomes more Maxwellian under the influence of numerical thermalisation as $N_{C}$ falls below about $100 \mathrm{Td}$.

The first two cases we have discussed are in the regime where inelastic collisions are important. Evidently, under these conditions, a modest number of particles suffices to produce a converged electron energy distribution function at almost all energies. At $10 \mathrm{Td}$, the case is somewhat different. Under these conditions, inelastic collisions are significant but not dominant in the electron energy balance - about $30 \%$ of the power is dissipated in inelastic collisions. Fig. 2 shows the variation of the electron mean energy with $N_{C}$ for this case (note that the data for this value of $E / N$ are scaled up by a factor 5). There is a much more significant variation of the mean energy with $N_{C}$, and a much larger number of particles $\left(N_{C} \sim 4000\right)$ is required to achieve a regime where the energy distribution function is converged. Fig. 5 shows that even with $N_{C}=4096$, the energy distribution is perceptibly too Maxwellian - there are more low energy electrons and more high energy electrons than are found in Monte Carlo simulations.

The final example deals with $E / N=1 \mathrm{Td}$, where inelastic processes are not important. In this case, the dependence of the mean energy on $N_{C}$ has changed, as fig. 2 shows (note that the data for this value of $E / N$ are scaled up by a factor 20). The mean energy slowly falls as $N_{C}$ increases. This small variation of the mean energy reflects a much larger change in the electron energy distribution functions, which are significantly too Maxwellian for any $N_{C} \lesssim 10^{4}$, as fig. 6 shows.

\section{Discussion and Conclusions}

The simulation results presented in the previous section show that the number of particles per cell that is required to obtain a converged electron energy distribution is a strong function of the electric field. For $E / N \gg 10 \mathrm{Td}, N_{C} \gtrsim 100$ appears sufficient, but for $E / N \lesssim 10 \mathrm{Td}$ a much larger number is needed. At $E / N \sim 1 \mathrm{Td}$, the number of particles needed to obtain a fully converged electron energy distribution function verges on the impractical. In all cases, the consequence of having too few particles is that the electron energy distribution function becomes too Maxwellian. Under the conditions discussed here, this means that the number of low energy electrons and the number of high energy electrons is greater than should be the case. In general, the nonphysical extension of the high energy tail of the distribution function probably has more serious consequences. These include overestimation of the rates of inelastic collision processes, and overestimation of the rate of untrapping of electrons near null points in the electric field, as occurs in the negative glow of some direct current discharges and in the bulk plasma of oscillating current discharges. Coulomb collisions may also be important under these conditions, and may limit the number of particles that are needed to ensure that physical effects dominate numerical phenomena [10].

These results apply to a specific set of calculations in helium, but they point to 
more general conclusions. If the main source of error in particle-in-cell simulations is velocity space diffusion, then a relevant accuracy criterion will be found by comparing the physical velocity space diffusion flux with its numerical counterpart, and choosing numerical parameters to ensure that the former is much greater than the latter. In practice, we usually lack the information to make such a comparison, especially a priori, in advance of actual computations. However, there is in practice a strong relationship between the electron temperature and the physical velocity space flux. When inelastic scattering is important, electrons are scattered out of the tail of the distribution very fast, and this scattering must be balanced by a relatively large velocity space flux at all energies where there is any significant density of electrons. Consequently, when there is an electron mean energy comparable to the inelastic threshold, there must also be a large velocity space flux, and a relatively small number of particles will be acceptable. In the opposite extreme, when elastic collisions dominate, the rate of scattering from the tail of the distribution will be relatively small, and will be balanced by a relatively small velocity space flux. In this case, one will need a relatively large number of particles to ensure that the numerical velocity space flux is small compared with the physical flux. The aim of the calculations presented above is to quantify the "relatively small" and "relatively large" number of particles referred to in these arguments. By this quantification, we seek to offer general guidance as to the number of particles likely to be required in any given context, either for the electron energy distribution function in general, or for groups of electrons within a larger distribution. (We note that only in the presence of non-local electron kinetics can there be such detached groups of electrons, such as are discussed in $[9,8,10]$.) The main indicator for the number of particles is consequently the ratio of the mean energy of the relevant electrons to the inelastic threshold, for the reasons just discussed. The calculations presented here refer to a specific ratio of the collision frequency to the plasma frequency. However, we have elsewhere investigated the dependence of the numerical velocity space fluxes on the collision frequency, which will indicate the relevant scaling to other situations. Of course, many other factors specific to a given situation may also be significant (the presence or absence of a Ramsauer minimum, for instance), but when the number of particles required can vary by three orders of magnitude, any advance information will be of value. The computations presented here give some such preliminary indication. The conclusion from such indications may sometimes be disagreeable, in that, especially for two- or three-dimensional simulations, it may be determined that a fully converged simulation cannot practically be achieved.

\section{Acknowledgements}

This work was supported by Science Foundation Ireland under grant numbers 07/IN.1/I907 and 08/SRC/I1411. 


\section{References}

[1] C. K. Birdsall and A. B. Langdon. Plasma Physics via Computer Simulation. McGraw-Hill, New York, 1985.

[2] R. W. Hockney and J. W. Eastwood. Computer Simulation using Particles. Adam Hilger, Bristol, 1988.

[3] C. K. Birdsall. Particle-in-cell charged-particle simulations, plus Monte Carlo collisions with neutral atoms, PIC-MCC. IEEE Trans. Plasma Sci., 19(2):65-85, April 1991.

[4] M. M. Turner. Collisionless heating in radio-frequency discharges: a review. J. Phys. D: Appl. Phys., 42:194008, 2009.

[5] Yuri P. Raizer. Gas Discharge Physics. Springer, Berlin, 1991.

[6] U. Kortshagen, C. Busch, and L. D. Tsendin. On simplifying approaches to the solution of the Boltzmann equation in spatially inhomogeneous plasmas. Plasma Sources Sci. Technol., 5:1-17, 1996.

[7] M. J. Kushner. Hybrid modelling of low temperature plasmas for fundamental investigations and equipment design. J. Phys. D: Appl. Phys., 42(19):194013, 2009.

[8] V. Vahedi, G. DiPeso, C. K. Birdsall, M. A. Lieberman, and T. D. Rognlien. Capacitive RF discharges modelled by particle-in-cell Monte Carlo simulation. I. analysis of numerical techniques. Plasma Sources Sci. Technol., 2:261-272, 1993.

[9] V. A. Godyak and R. B. Piejak. Abnormally low electron temperature and heatingmode transition in a low-pressure rf discharge at $13.56 \mathrm{MHz}$. Phys. Rev. Lett., 65 (8):996-999, August 1990.

[10] M. M. Turner. Kinetic properties of particle-in-cell simulations compromised by Monte Carlo collisions. Phys. Plasmas, 13(3):033506, March 2006.

[11] J. L. Pack, R. E. Voshall, A. V. Phelps, and L. E. Kline. Longitudinal electron diffusion coefficients in gases: Noble gases. J. Appl. Phys., 71(11):5363-5371, June 1992.

[12] J. E. Lawler, E. A. Den Hartog, and W. N. G. Hitchon. Power balance of negativeglow electrons. Phys. Rev. A, 43(8):4427-4437, April 1991. 


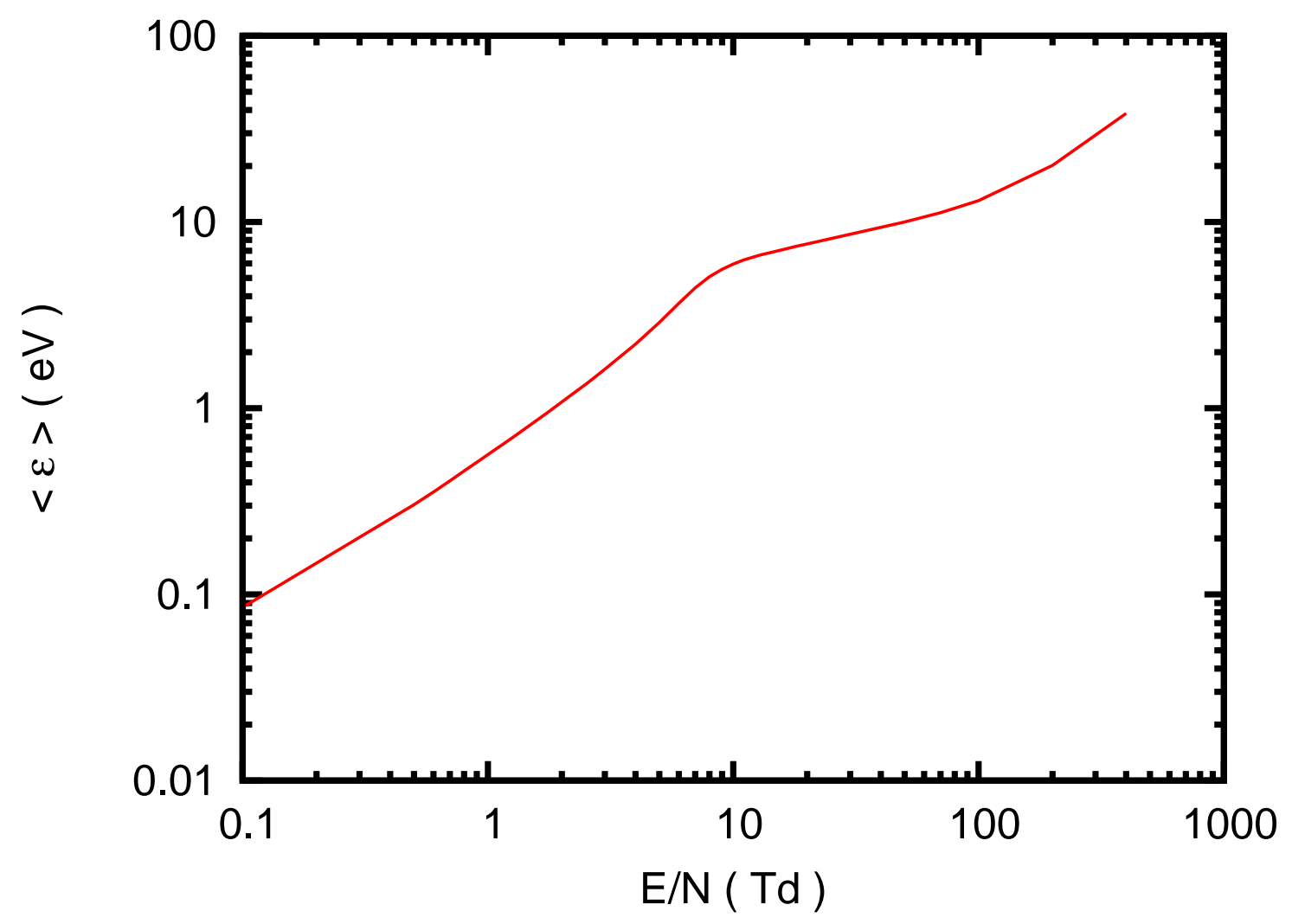

Figure 1. The electron mean energy $\langle\varepsilon\rangle$ as a function of the reduced electric field for electrons in helium, showing the range of values considered in this paper. 


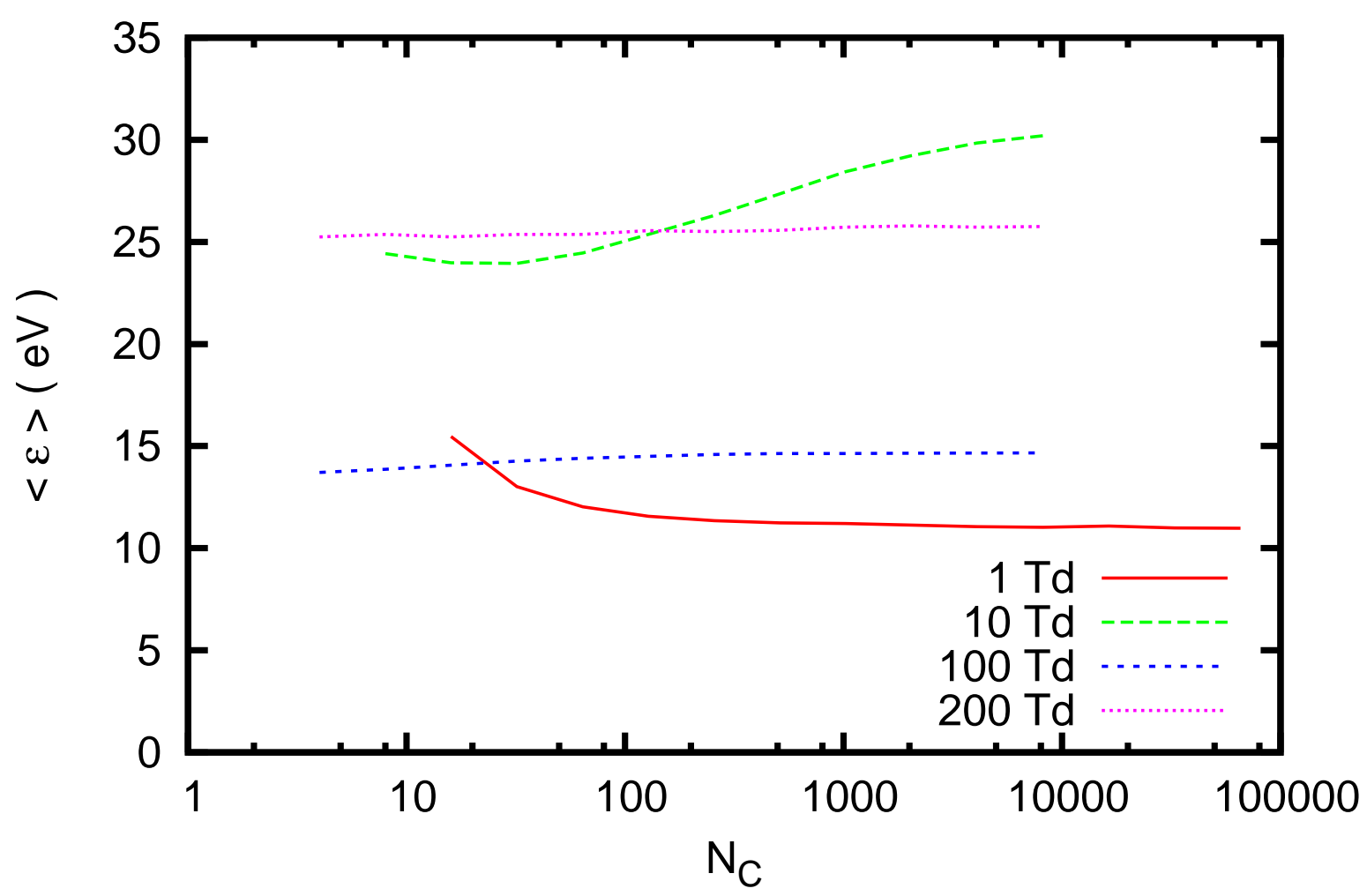

Figure 2. The electron mean energy calculated using a particle-in-cell simulation with varying number of particles per cell, $N_{C}$, for the four reduced electric field strengths considered in this paper. Other numerical parameters are kept constant. Note that, for clarity of presentation, the data for $1 \mathrm{Td}$ have been multiplied by a factor of 20 , and the data for $10 \mathrm{Td}$ have been multipled by a factor of 5 . 


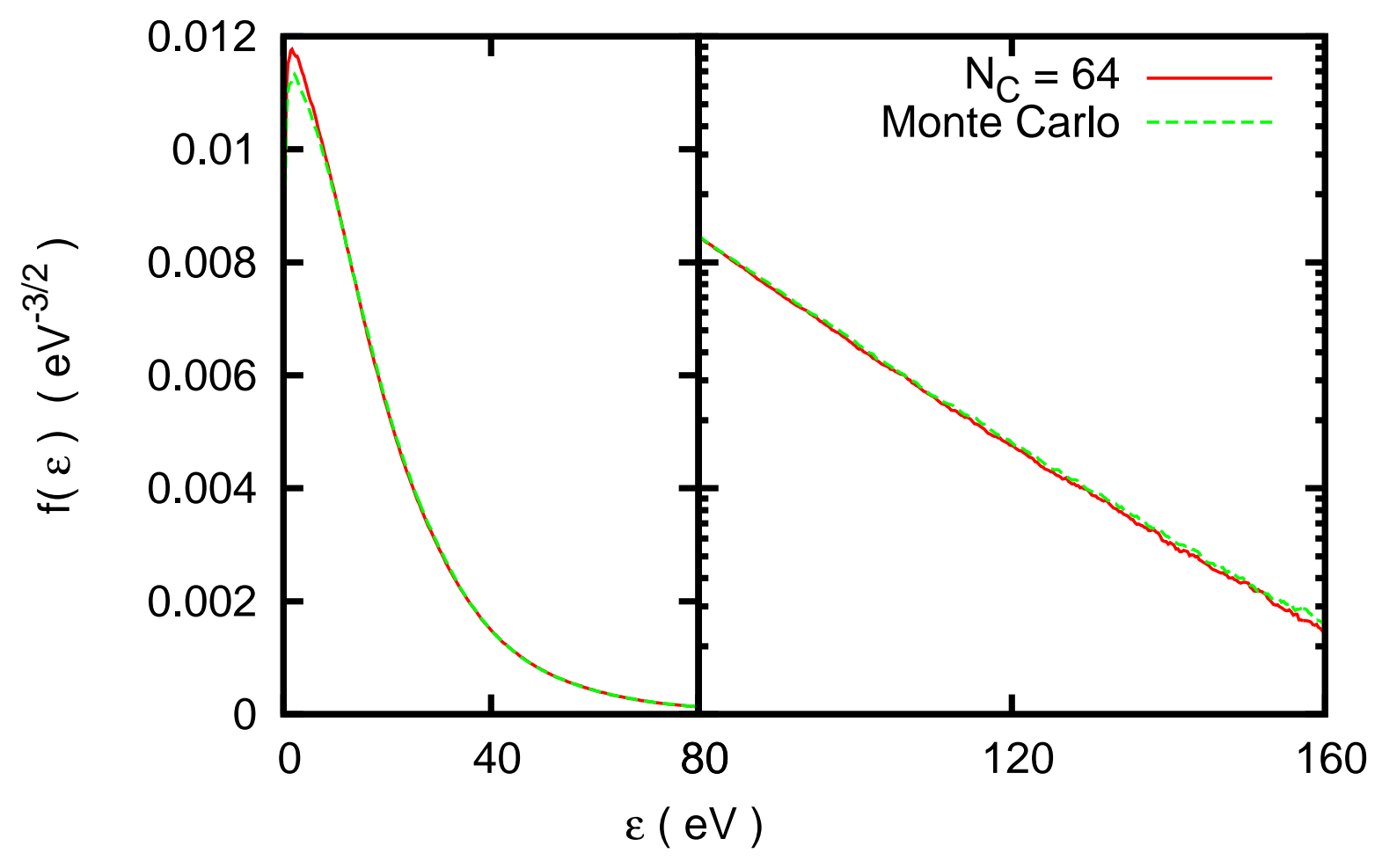

Figure 3. The electron energy distribution function at a reduced electric field strength of $E / N=200 \mathrm{Td}$, calculated using a particle-in-cell simulation and a Monte Carlo simulation. The same distribution functions are show in each half of the figure, but on the left the vertical scale is linear, and on the right it is logarithmic. 


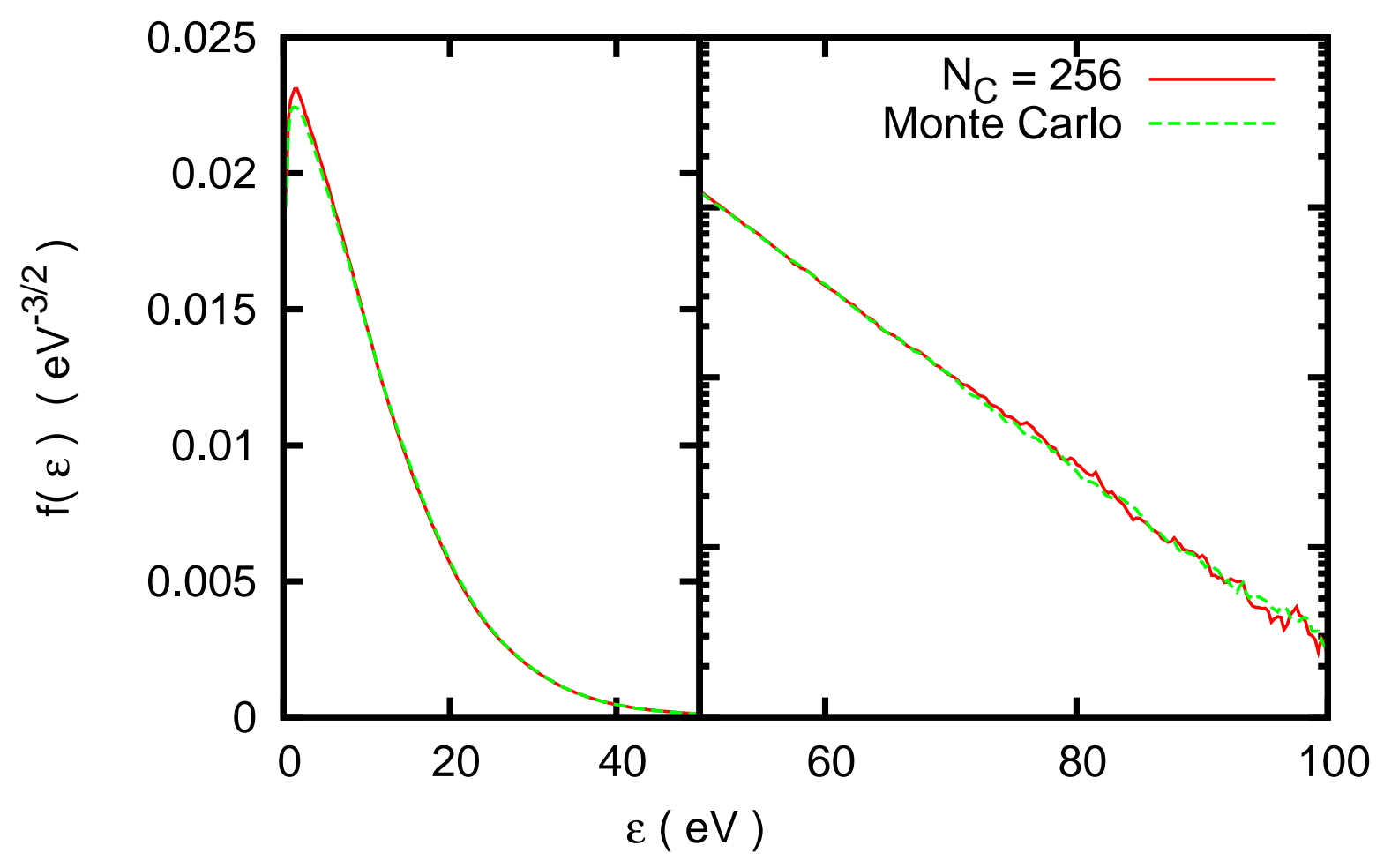

Figure 4. The electron energy distribution function at a reduced electric field strength of $E / N=100 \mathrm{Td}$, calculated and displayed as described in the caption for fig. 3 


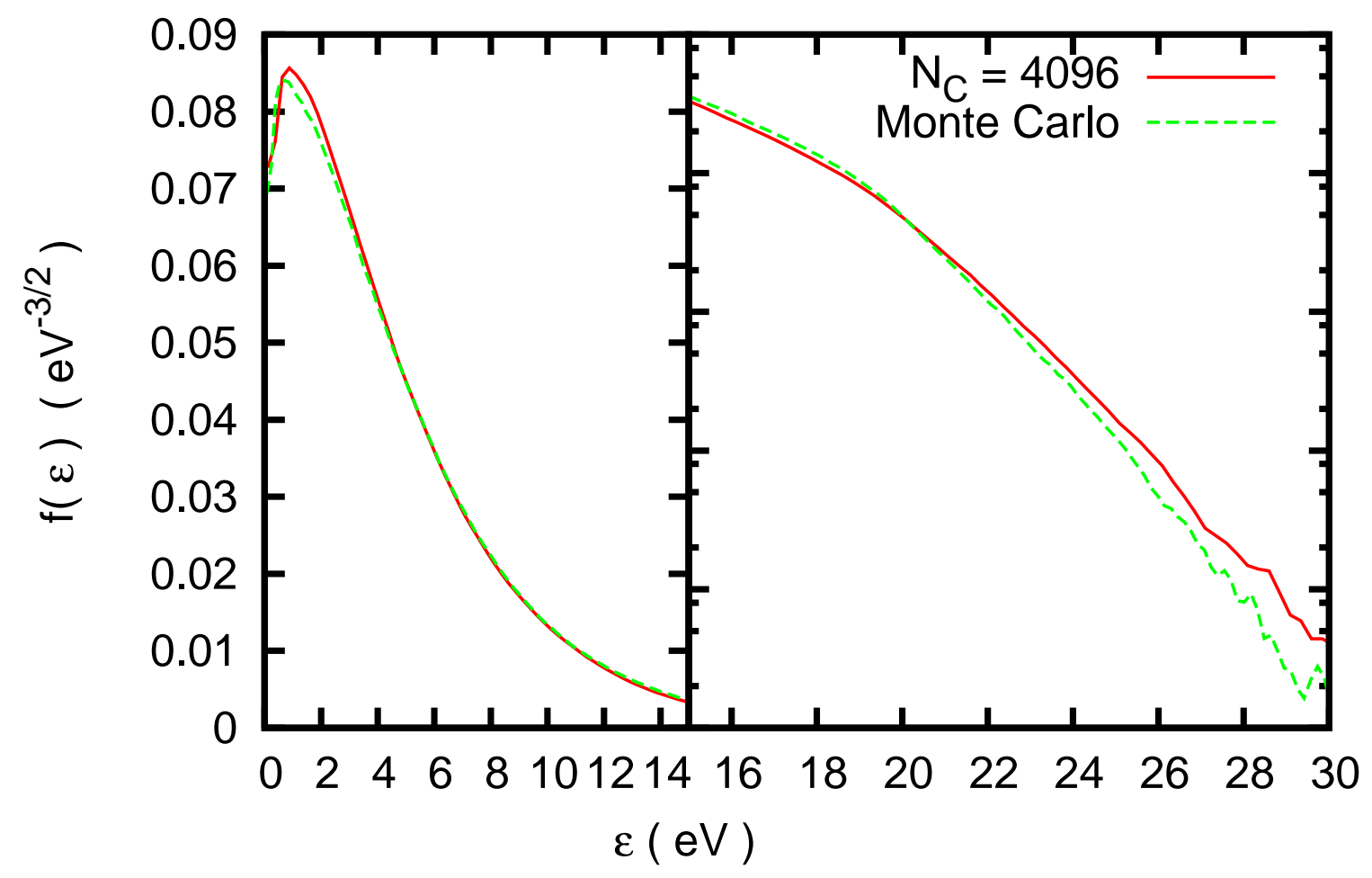

Figure 5. The electron energy distribution function at a reduced electric field strength of $E / N=10 \mathrm{Td}$, calculated and displayed as described in the caption for fig. 3 


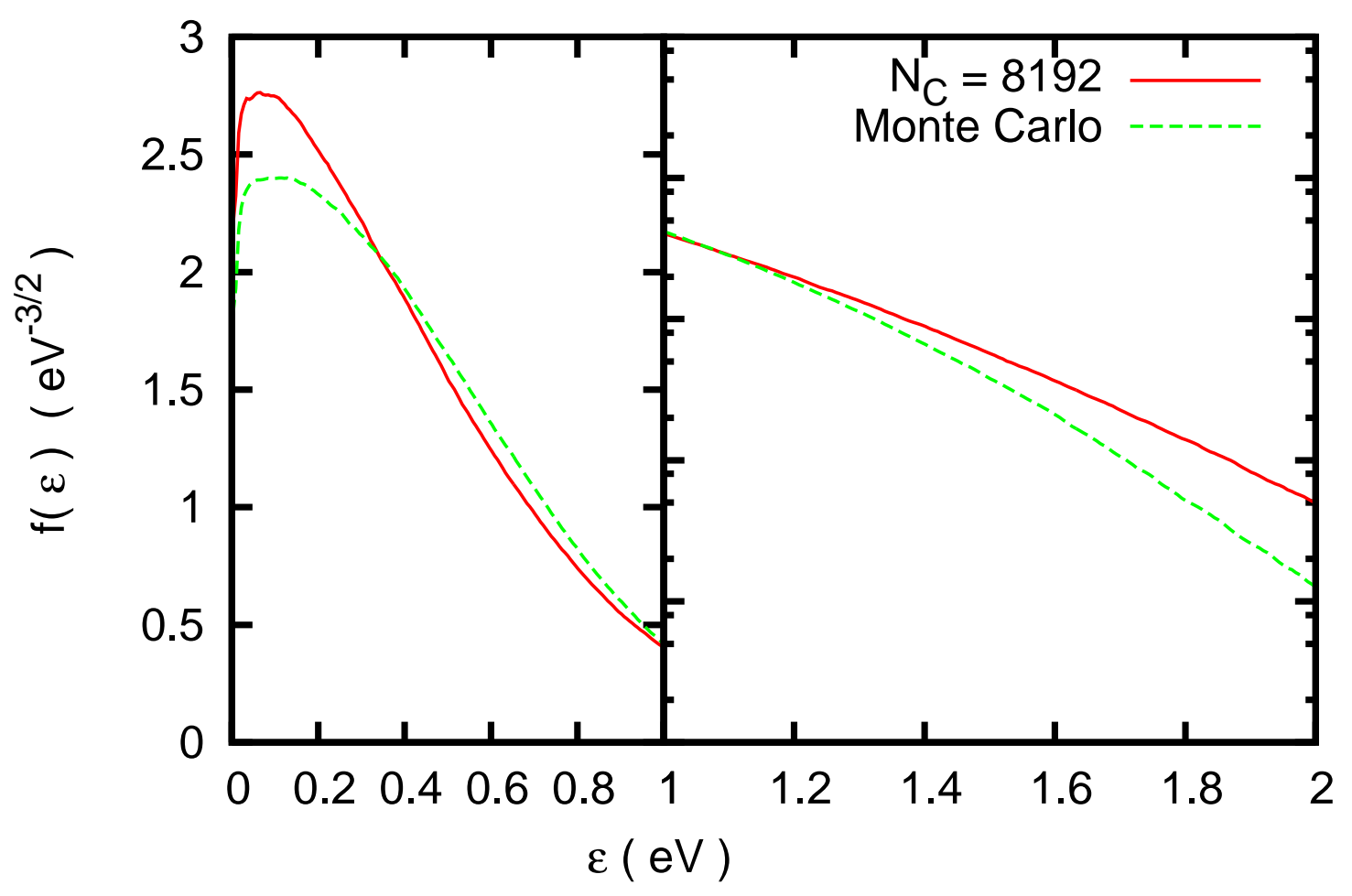

Figure 6. The electron energy distribution function at a reduced electric field strength of $E / N=1 \mathrm{Td}$, calculated and displayed as described in the caption for fig. 3 